\title{
Uso de receptores monofrecuencia para mediciones geodésicas en sistema GNSS, en la región del Nordeste
}

Corvalán, Rubén E. ${ }^{\left({ }^{*}\right)(1)}$, Schaller, José O. ${ }^{\left({ }^{*}\right)(2)}$; Titiosky, Valeria S. ${ }^{\left({ }^{*}\right)(3)}$; Ferrari, Elvira E. ${ }^{()^{(*)(4)}}$

\section{RESUMEN}

El Sistema de Posicionamiento Global (GPS) es un sistema que tiene por objetivo la determinación de coordenadas espaciales de puntos respecto de un sistema de referencia mundial y su funcionamiento se basa en la idea clásica de triangulación. Para determinar la posición de un punto en el espacio, es suficiente conocer las distancias a tres puntos de coordenadas conocidas. E1 Posicionamiento Puntual es la manera más sencilla de usar el GPS; si solo se cuenta con un equipo económico (navegador) que sea capaz de observar el código (C/A o P), se puede obtener, a partir del mismo, la distancia satélite-receptor o, más exactamente, el tiempo en que la señal va desde el satélite al receptor. La existencia de los errores en éste método, cuya fuente son los

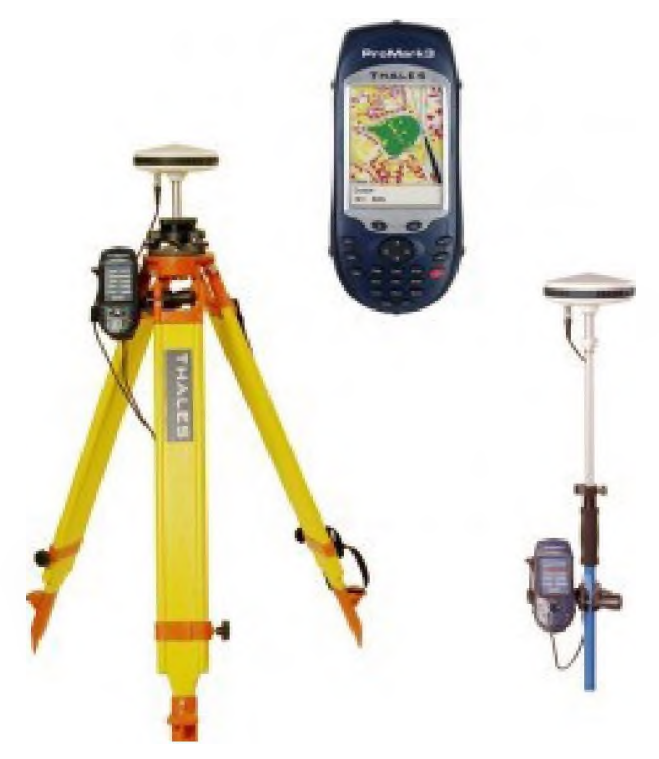

relojes, ionosfera, error en las efemérides, troposfera, llevó a desarrollar la técnica del Posicionamiento Diferencial, que consiste en medir simultáneamente con dos receptores GPS, uno de coordenadas conocidas

\footnotetext{
() Departamento de Agrimensura - Facultad de Ciencias Exactas y Naturales y Agrimensura (U.N.N.E.) - 9 de julio 1449,3400 , Corrientes

(1) Magister en Administración de Negocios - rubenpstt@yahoo.com.ar

(2) Agrimensor - joseschaller1234@gmail.com

(3) Especialista en Tecnología de Información Geográfica - vstitios@hotmail.com

(4) Especialista en Docencia Universidad - elviraeferrari@yahoo.com.ar
} 
que debe permanecer quieto y sobre el otro no existe restricción de movimiento. Esta técnica puede alcanzar, en el mejor de los casos, exactitudes milimétricas pero, cuando solo se cuentan con equipos económicos (monofecuencia), la técnica diferencial deja un residuo importante sobre la distancia relativa entre receptores. Los Mapas Ionosféricos provistos por los Modelos Ionosféricos mitigan el efecto que la ionósfera causa sobre el Posicionamiento Puntual y sobre las redes geodésicas, brindando la oportunidad de introducir correcciones por retardo ionosférico en el procesamiento de vectores en los receptores GPS denominados "navegadores" y de monofrecuencia. La contribución pretendida por el presente trabajo es determinar la eficacia de los mapas ionosféricos, en particular el generado por el modelo LPIM, aplicados al procesamiento de vectores observados con receptores GPS de monofrecuencia.

Palabras claves: GPS - Posicionamiento - Coordenadas - Monofrecuencia

\section{SUMMARY}

The Global Positioning System (GPS) is a system that aims to determine spatial coordinates of points with respect to a world reference system and its operation is based on the classic idea of triangulation. To determine the position of a point in space, it is sufficient to know the distances to three points of known coordinates. Point Positioning is the simplest way to use GPS; if you only bave an economic equipment (browser) that is able to observe the code $(C / A$ or $P)$, you can obtain from it the satelli- te-receiver distance or, more exactly, the time in which the signal goes from the satellite to the receiver. The existence of errors in this method, whose source are clocks, ionosphere, error in the ephemeris, troposphere, led to develop the $\mathrm{Di}_{\mathrm{i}}$ fferential Positioning technique that, consists of simultaneously measuring with two GPS receivers, one of known coordinates that must remain still and on the other there is no restriction of movement. This technique can reach, in the best of cases, millimeter accuracy but, when only economic equipment is available (monofequency), the differential technique leaves an important residue on the relative distance between receivers. The Ionosphore Maps provided by the Ionospheric Models mitigate the effect that the ionosphere causes on the Positioning Point and on the geodetic networks, providing the opportunity to introduce corrections by ionospheric delay in the processing of vectors in GPS receivers called "browsers" and monofrequency. The contribution sought by the present work is to determine the efficiency of the ionospheric maps, in particular the one generated by the LPIM model, applied to the processing of observed vectors with GPS monofrequency receivers.

Keywords: GPS - Positioning - Coordinates - Monofrequency

\section{El Sistema de Posicionamiento Glo-} bal (GPS) es un sistema de posición, tiempo y orientación, basado en la medición simultanea desde, por lo menos, cuatro satélites, de posición conocida en el sistema de referencia global denominado WGS84. La condición operacional del sistema invo- 


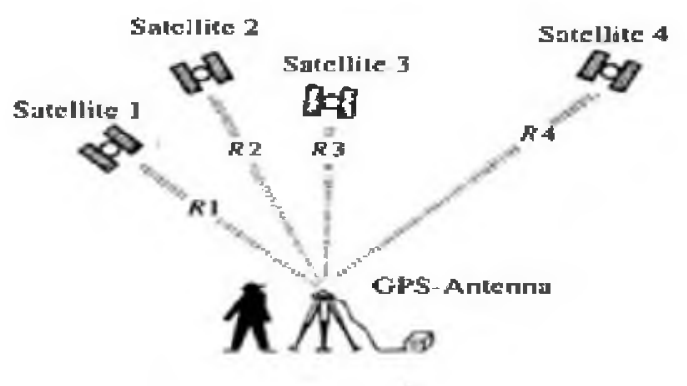

Figura 1: Método de posicionamiento

lucra la relación entre tres segmentos fundamentales: terrestre, espacial y usuario. La relación se establece mediante señales de radio.

Las distancias son medidas comparando la señal recibida desde el satélite con una réplica generada por el receptor. La diferencia de fase entre la señal recibida y la generada localmente es proporcional al tiempo de propagación (distancia Satélite/receptor). Como los receptores generan la señal local usando relojes de cuarzo, la diferencia de fase absorbe también sus inestabilidades. Observando un mínimo de cuatro satélites bien distribuidos en el cielo, el receptor puede calcular cuatro incógnitas: latitud, longitud, altura y corrección al reloj local.

El GPS fue diseñado como un sistema de navegación autónomo, y este sigue siendo el principal uso del sistema hoy en día. También existe una forma más sofisticada de uso donde es necesario contar con al menos otro receptor GPS.

Basa su funcionamiento en la idea clásica de triangulación (Figura 1). Cuando se conocen las distancias de varios objetos (de coordenadas conocidas) respecto de uno (de coordenadas desconocidas) es posible ubicar este último. Para realizar esto en el espacio son necesarios al menos tres distancias a lugar. Los objetos (tanto aquellos cuya posición es conocida como aquel cuya posición se desconoce) no tienen por qué estar fijos en el espacio, los satélites GPS se encuentran permanentemente en movimiento. Esta característica hace que, si se observa por un tiempo prolongado, cambie la geometría del problema y por lo tanto mejore la resolución de la posición. Otra forma de mejorar la exactitud es usando los distintos observables (códigos C/A y $\mathrm{P}$ ) o la fase de las frecuencias portadoras (L1 y L2). Esto da lugar a distintas estrategias de uso del GPS.

La portadora L1 es transmitida a una frecuencia de $1575.42 \mathrm{MHz}(10.23 \times 154)$ con una

longitud de onda de $19.05 \mathrm{~cm}$; la portadora L2 es transmitida a una frecuencia de $1227.60 \mathrm{MHz}(10.23 \times 120)$ con una longitud de onda de $24.45 \mathrm{~cm}$; el Código C/A o Código de Adquisición Gruesa (libre adquisición) modula a una frecuencia de $1.023 \mathrm{MHz}(10.23 / 10)$, tiene una duración de un milisegundo, su longitud de onda es de aproximadamente $300 \mathrm{~m}$ y se transmite actualmente sólo por medio de la frecuencia portadora L1; el Código $\mathrm{P}$ o Código de Precisión modula a una frecuencia de $10.23 \mathrm{MHz}$, su secuencia es de 267 días y su longitud de onda es de $29.31 \mathrm{~cm}$. (Se les ha asignado a los distintos satélites porciones de siete días y como resultado de ello, todos los satélites pueden trasmitir en la misma frecuencia y ser identificados por sus segmentos únicos de una semana) 
El Posicionamiento Puntual es la manera más sencilla de usar el GPS. Si solo se cuenta con un equipo económico (navegador) que sea capaz de observar el código $(\mathrm{C} / \mathrm{A}$ o $\mathrm{P})$ se puede obtener, a partir del mismo, la distancia satélite-receptor o, más exactamente, el tiempo en que la señal va desde el satélite al receptor. Como la señal GPS transmite también la posición (efemérides) de los satélites, es posible ubicar al receptor GPS.

Si el usuario conoce su altitud, tres satélites son suficientes. Las posiciones futuras de los satélites y el estado de sus relojes son estimados a partir de mediciones de distancias similares a las efectuadas por cualquier usuario, realizadas desde las estaciones de rastreo distribuidas en el globo. Usando complejos algoritmos de ajuste orbital, la estación maestra ubicada en la base Falcón de la Fuerza Aérea de Colorado Springs, calcula las orbitas y predice las posiciones futuras de los satélites y el estado de sus relojes.

Las posibles fuentes de error (Figura 2 y Figura 3) pueden deberse a:

- Los satélites: error del reloj y error orbital.

- Al medio de propagación de la señal: refracción ionosferica y troposférica, perdida de ciclos y multicamino.

- A los receptores: error del reloj, variación del centro de la antena, Multipath o multicamino, estación error en las coordenadas.

- A la posición de los receptores.

- Dilución de la precisión.

- Precisión con que se correlacionan las portadoras del satélite y el receptor (ruido pseudoaleatorio).

- Imprecisión de las coordenadas de los satélites.

- Desincronización de los relojes del satélite y del receptor con respecto al tiempo GPS.

- Error de correlación de ruido, producido en el receptor a producir la alineación de Orden del 1\% de la longitud de onda fase.

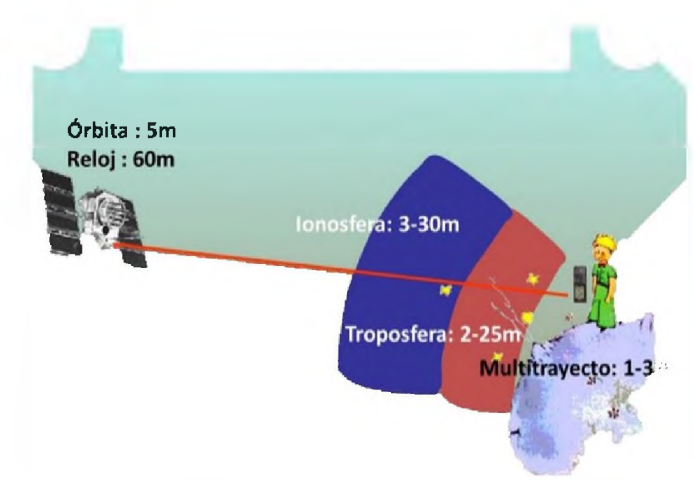

Figura 2. Fuentes de error

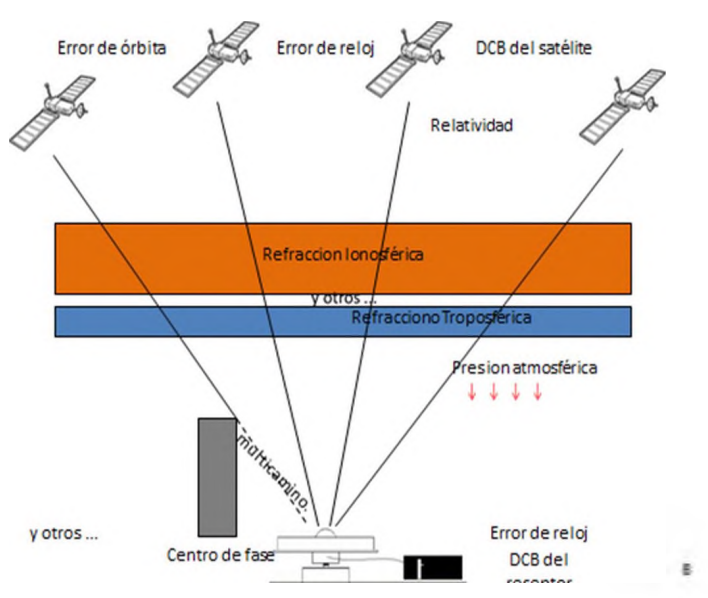

Figura 3. Fuentes de error

La existencia de los errores en los relojes, ionosfera, error en las efemérides, tro- 
posfera, entre otros, en el uso del GPS, llevó a desarrollar la técnica del Posicionamiento Diferencial. Consiste en medir simultáneamente con dos receptores GPS: uno de coordenadas conocidas que debe permanecer quieto; sobre el otro no existe restricción de movimiento. $\mathrm{La}$ adecuada combinación de las señales procedentes de los distintos receptores hace posible eliminar gran parte de los mismos y es capaz de brindar la posición del receptor de coordenadas desconocidas en función de la otra. El método diferencial fue, además, el primero que permitió el uso de las fases (cuya resolución es 1000 veces superior a la de los códigos) como información útil para posicionarse. Esto es debido a que, como en otras técnicas interferométricas, si se usan señales senoidales puras (como las portadoras L1 y L2), solo es posible medir el último ciclo no completo de la señal, pero se desconoce la cantidad de ciclos enteros que hay entre cada satélite y el receptor GPS. Esta limitación hace que las fases no sean usadas en el posicionamiento puntual. Esta técnica puede alcanzar, en el mejor de los casos, exactitudes milimétricas y, justamente por eso, requiere de un correcto modelado de todos los sistematismos. Cuando se cuenta con receptores que observan sobre L1 y L2 es posible realizar esto; cuando solo se cuentan con equipos económicos (monofecuencia), la técnica diferencial deja un residuo importante sobre la distancia relativa entre receptores.

La atmósfera del planeta Tierra constituye uno de los sistemas físicos más complejos conocidos por el hombre (Figura 4). Se la puede definir como una mezcla de gases que interactúa química, dinámica y electromagnéticamente con el medio que se extiende desde la superficie terrestre hasta los alrededores del Sol. Al tratarse de una mezcla de gases, puede ser estudiada y clasificada de acuerdo a diferentes parámetros (o cantidades físicas) de referencia, como la temperatura y la composición química. Se la puede dividir en dos grandes regiones: la atmósfera neutra, que comprende la región que se extiende desde la superficie terrestre hasta los $50 \mathrm{~km}$ de altura aproximadamente, y la ionosfera, región de la atmósfera comprendida entre los 50-60 km de altura y los $1000 \mathrm{~km}$ de altura, aproximadamente. Debido a sus propiedades electromagnéticas, la ionosfera ha fascinado a los científicos desde principios del siglo XX.

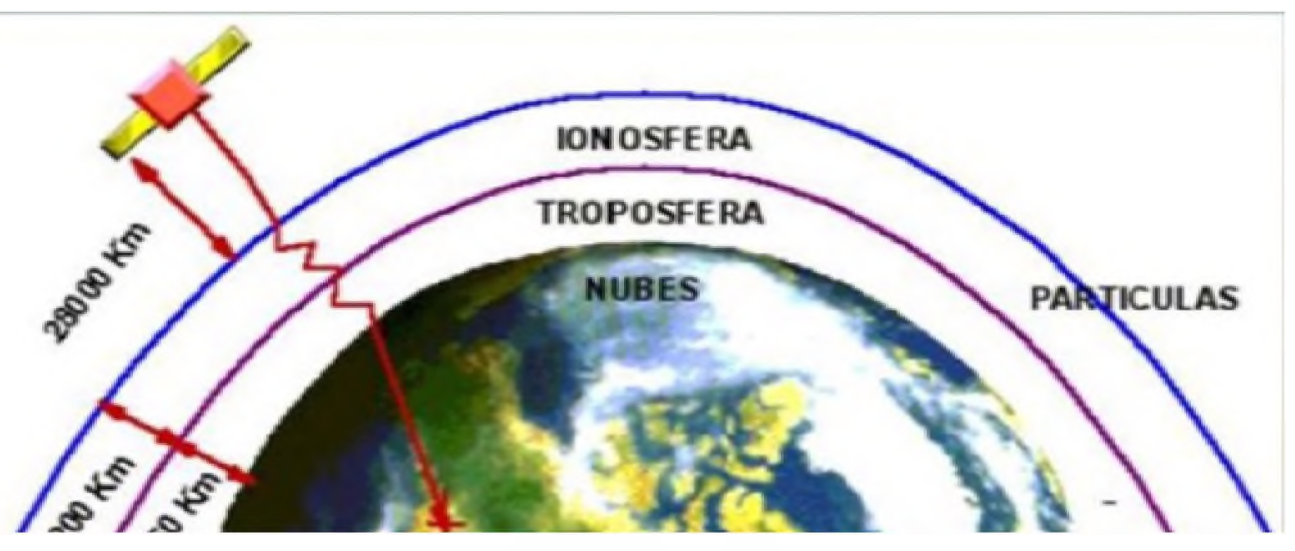

Figura 4. La atmosfera 
La señal GPS, en su camino SATÉLITE-RECEPTOR, se propaga a través de regiones de la atmósfera de naturaleza diversa y variable estado y experimenta modificaciones de diversas especies. Las perturbaciones de producen en la dirección de propagación en la velocidad y la fuerza de la señal.

El impacto que produce en los resultados de las observaciones exceden las tolerancias del sistema (1 metro a 100 metros). Se corrigen por medición directa o modelación.

La precisión alcanzada dependerá:

- Del tipo de receptor que se utilice y del campo de estudio.

- Los receptores geodésicos alcanzan precisiones del orden del $\mathrm{cm}$ en la determinación de la posición.

- Los navegadores tienen una precisión de 5 a 15 metros.
La precisión de la posición relativa resulta afectada en 1ppm que significa $1 \mathrm{~cm}$ en $10 \mathrm{~km}$. Error de la base es $1 \mathrm{ppm}$ cada 10 $m$ de error en las coordenadas de partida.

$\mathrm{La}$ ionosfera es el mayor agente de error en la determinación de coordenadas por el método de posicionamiento absoluto siendo este método uno de los más empleado por los usuarios de GPS, con fines geodésicos.

La ionosfera es una sección de la alta atmósfera que se encuentra lo suficientemente ionozada como para afectar a las ondas de radio que por ellas se propagan. Esta ionización está provocada por la radiación ultravioleta que proviene del sol y por partículas de alta energía que se precipitan desde el espacio. Por esta característica, la región sufre la influencia de campos magnéticos y eléctricos que, recíprocamente, son perturbados por ella. La ionosfera es la región

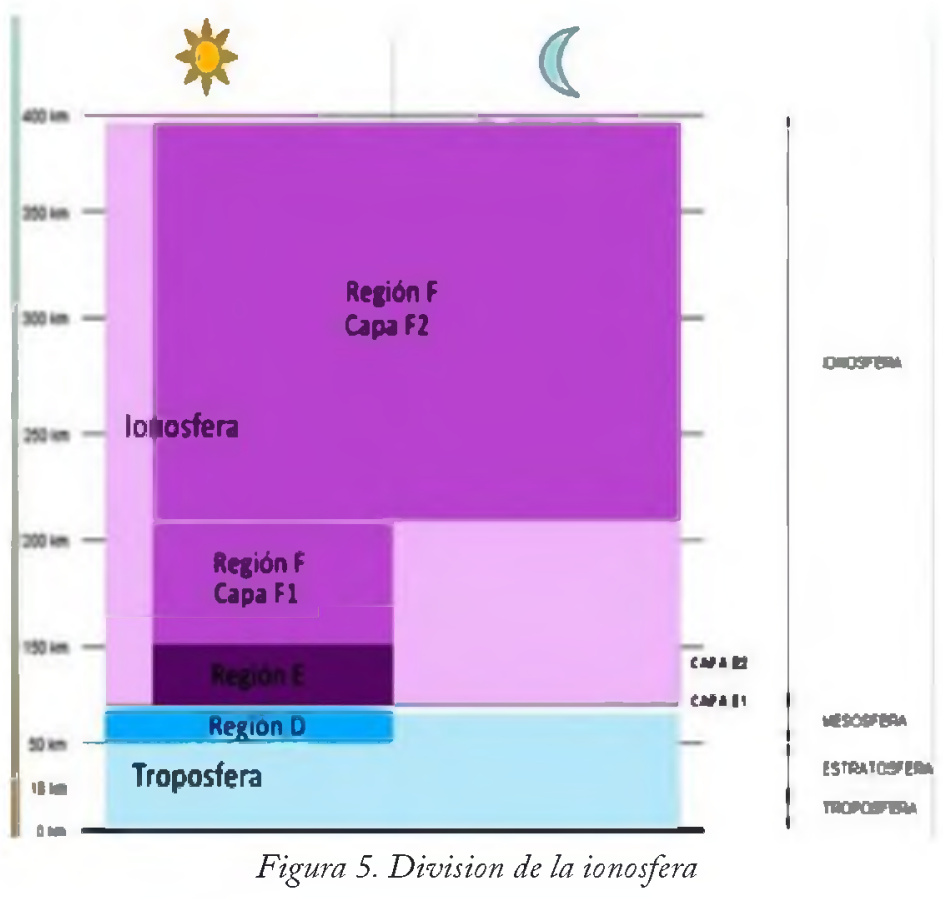


que acopla o vincula la magnetosfera y la atmósfera.

La ionosfera puede ser dividida en cuatro capas: D, E, F1 y F2 (Figura 5). La región $\mathrm{D}$ se encuentra entre los 65 y $90 \mathrm{ki}-$ lómetros y su ionización relativamente baja absorbe principalmente frecuencias de radio altas. La región $\mathrm{E}$ está localizada entre los 90 y 150 kilómetros conteniendo principalmente iones de oxigeno $\mathrm{O} 2+$. Las regiones $\mathrm{F}$ están sobre los 150 kilómetros y son las más importantes desde el punto de vista de las comunicaciones de radio. Particularmente la región $\mathrm{F} 2$ donde la concentración de electrones presenta un máximo pronunciado, situación que más adelante permitirá aproximar a la ionosfera como una capa de espesor infinitesimal. En la parte inferior de la región $\mathrm{F}$ predominan los iones $\mathrm{NO}+$, mientras que en la superior lo hacen los $\mathrm{O}+$. Más allá de la región $\mathrm{F}$, la densidad electrónica decrece hasta perder influencia en la perturbación de señales electromagnéticas; esta influencia todavía puede ser notable en la plasmasfera, región que se encuentra por encima de los $2000 \mathrm{~km}$

La ionosfera, que retrasa la señal en una cantidad variable y desconocida, es la principal fuente de error del método. La única manera de eliminarla sería contando con navegadores que midan sobre el código $\mathrm{C} / \mathrm{A}$ y $\mathrm{P}$, lo que, si bien es posible, no resulta práctico por motivos económicos.

El hecho de que esté constituida por partículas eléctricamente cargadas (iones y electrones, de allí su nombre), la convierte en un instrumento de vital importancia para las radio-comunicaciones. Tanto es así, que muchos de los radares que actualmente se implementan para su estudio fueron originalmente utilizados como radares de comunicaciones militares durante la Segunda Guerra Mundial. Sin embargo, la investigación ionosférica con fines puramente científicos nunca cesó; y, si bien hace más de 80 años que la ionosfera es objeto de análisis, su estudio ha cobrado especial importancia durante el transcurso de las últimas décadas.

Fue durante la década de 1990 (en el año 1994 comienza a operar el Sistema de Posicionamiento Global, o GPS por su sigla en inglés) y la primera década del siglo XXI que el conocimiento de las características físicas de la ionosfera se convirtió en una necesidad de extrema importancia, puesto que la capacidad de predicción de los fenómenos ionosféricos resulta esencial para la optimización de los sistemas de radiocomunicaciones y los de navegación global basados en satélites. Esta necesidad de conocimiento y capacidad de predicción ionosféricas requiere de instrumentación de alta sensibilidad y confiabilidad, así como también, de modelos que, basados en hipótesis teóricas, incorporen los datos medidos por los instrumentos disponibles para mejorar la descripción de la física de la ionosfera, y de esta manera, logren una predicción precisa de los fenómenos que en ella ocurren, a partir de la verificación y/o corrección de las hipótesis establecidas a priori. En consecuencia, durante las últimas décadas, gran cantidad de instrumentos han sido desarrollados, mejorados e instalados alrededor del globo y a bordo de satélites artificiales. Esto, conjuntamente con el crecimiento exponen- 
cial de la tecnología, ha permitido que hoy en día se cuente con una cantidad y variedad de datos ionosféricos sin precedentes. $Y$ es justamente esta variedad y disponibilidad de datos observacionales, la que coloca a los modelos ionosféricos semi-empíricos en la vanguardia del estudio de la ionosfera y sus propiedades físicas (Figura 6).

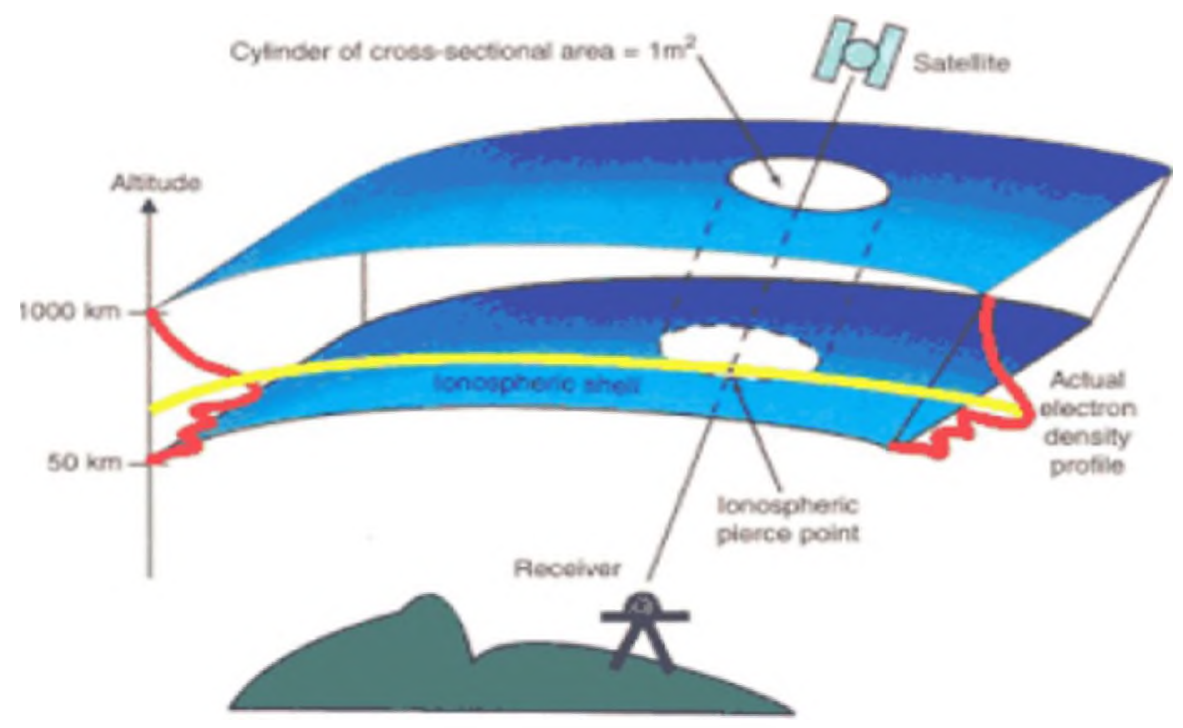

Figura 6. Señal del satelite

Correcciones ionosféricas en GNSS

El principal motivo por el que el sistema GPS transmite en 2 frecuencias es para permitir la eliminación de efectos vinculados con la ionosfera

$$
\begin{gathered}
\mathrm{P} 1=\mathrm{d}+\frac{40,3 \mathrm{TEC}}{\mathrm{f} 1^{2}} \\
\mathrm{P} 2=\mathrm{d}+\frac{40,3 \mathrm{TEC}}{\mathrm{f} 2^{2}}
\end{gathered}
$$

$\mathrm{P} 1=$ Distancia medida con frecuencia f1

$\mathrm{d}=$ distancia verdadera

$\mathrm{P} 2=$ Distancia medida con frecuencia $f 2$

$\mathrm{TEC}=$ Contenido total de electrones en el camino de la señal

El retardo ionosférico en las medidas GNSS puede cancelarse usando la combinación "libre de ionosfera"

$$
d=\frac{f_{1 . P_{1}}^{2}+f_{2}^{2} * p_{2}}{f_{1}^{2}+f_{2}^{2}}
$$

En los receptores que leen una sola frecuencia esa combinación no es posible y para reducir el retardo ionosferico hay que introducir, de alguna manera, en los archivos registrados, el valor del TEC antes del procesamiento del vector.

Como fuente de este dato, existen varias instituciones que lo proveen, como muestra la Figura 7.

Existen mapas ionosféricos elaborados por el modelo LPIM, desarrollado por la Universidad Nacional de La Plata y utilizado por el Centro de Análisis Ionosférico 
- IGS $\rightarrow$ InternationalGNSS Service
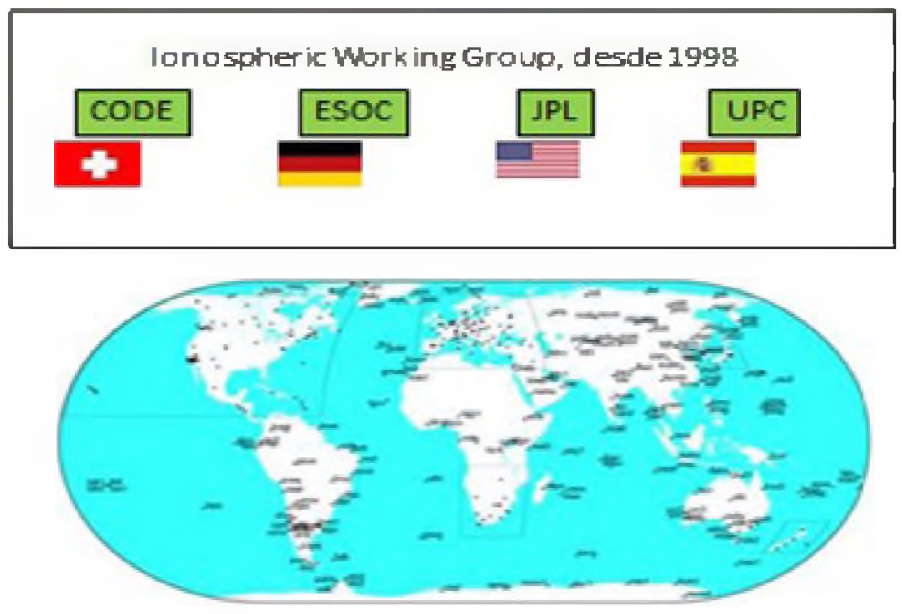

Figura 7. Mapas disponibles

de SIRGAS para producir mapas de VTEC para América Latina. (Figura 8)

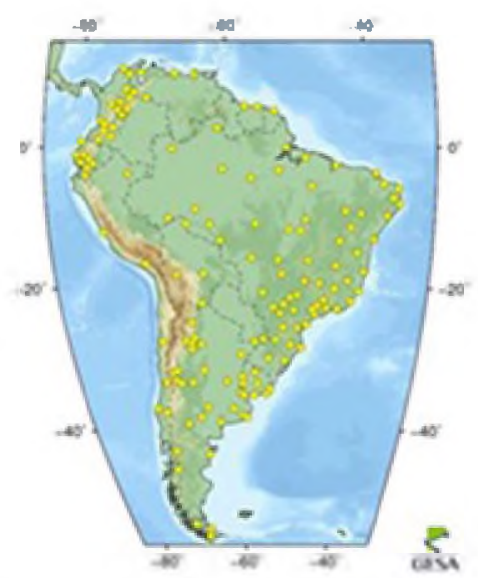

Figura 8. LPIM (La Plata Ionospheric)

Los valores de TEC se presentan en forma de Mapas (Figura 9) y en forma de grillas numéricas (Tabla 1). http://cplat. fcaglp.unlp.edu.ar/products/iono/etc/

Entonces, se podría elaborar un procedimiento normatizado para introducir los datos de los modelos ionosfericos en los programas de cálculo de receptores de monofrecuencia de distintos fabricantes.

En la región, encontramos en forma operable, un número importante de receptores simple frecuencia, de acuerdo a una encuesta realizada en organismos públicos y usuarios particulares, que son destinatarios del beneficio que acarrearía, en precisión y alcance de sus equipos, con la introducción de las correcciones por retardo ionosférico.

Como base se comienza con el estudio de los formatos de intercambio RINEX e IONEX. Y el procedimiento base es el graficado en la Figura 10. 


\begin{tabular}{|c|c|c|}
\hline LONGITLD & LATITUD & TECU \\
\hline 251.00 & -60.00 & 37.22 \\
\hline 252.00 & -60.00 & 35.89 \\
\hline 253.00 & -60.00 & 34.65 \\
\hline 254.00 & -60.00 & 33.50 \\
\hline 255.00 & -60.00 & 32.43 \\
\hline 256.00 & -60.00 & 31.45 \\
\hline 257.00 & -60.00 & 30.55 \\
\hline 258.00 & -60.00 & 29.72 \\
\hline 259.00 & -60.00 & 28.97 \\
\hline 260.00 & -60.00 & 28.29 \\
\hline $26: .00$ & -60.00 & 27.59 \\
\hline 262.00 & -60.00 & 26.94 \\
\hline 263.00 & -60.00 & 26.35 \\
\hline 264.00 & -60.00 & 25.82 \\
\hline 265,00 & -60.00 & 25.33 \\
\hline 266.00 & -60.00 & 24.88 \\
\hline 267.00 & -60.00 & 24.47 \\
\hline 268.00 & -60.00 & 24.10 \\
\hline 269.00 & -60.00 & 23.75 \\
\hline 270,00 & -60.00 & 23.44 \\
\hline 271.00 & -60.00 & 23.18 \\
\hline 272.00 & -60.00 & 22.94 \\
\hline 273.00 & -60.00 & 22.71 \\
\hline 274.00 & -60.00 & 22.49 \\
\hline 275.00 & -60.00 & 22.28 \\
\hline 276.00 & -60.00 & 22.06 \\
\hline 277.00 & -60.00 & 21.84 \\
\hline
\end{tabular}

\begin{tabular}{|c|c|c|}
\hline LONGITLD & LATITUD & TECU \\
\hline 277.00 & -60.00 & 21.84 \\
\hline 278.00 & -60.00 & 21.62 \\
\hline 279.00 & -60.00 & 21.39 \\
\hline 280.00 & -60.00 & 21.16 \\
\hline 281.00 & -60.00 & 20.92 \\
\hline 282.00 & -60.00 & 20.67 \\
\hline 283.00 & -60.00 & 20.42 \\
\hline 284.00 & -60.00 & 20.17 \\
\hline 285.00 & -60.00 & $19.9 \mathrm{~L}$ \\
\hline 286.00 & -60.00 & 19.65 \\
\hline 287.00 & -60.00 & 19.40 \\
\hline 288.010 & -60.00 & 19.14 \\
\hline 289.00 & -60.00 & 18.89 \\
\hline 290.00 & -60.00 & 18.64 \\
\hline 291.00 & -60.00 & 18.43 \\
\hline 292.00 & -60.00 & 18.22 \\
\hline 293.00 & -60.00 & 18.02 \\
\hline 294.00 & -60.00 & 17.83 \\
\hline 295.00 & -60.00 & 17.64 \\
\hline 296.00 & -60.00 & 17.45 \\
\hline 297.00 & -60.00 & 17.28 \\
\hline 298.00 & -60.00 & 17.10 \\
\hline 299.00 & -60.00 & 16.93 \\
\hline 300.00 & -60.00 & 16.75 \\
\hline 301.00 & -60.00 & 16.52 \\
\hline 302.00 & -60.00 & 16.29 \\
\hline 303.00 & -60.00 & 16.05 \\
\hline
\end{tabular}

Tabla 1. LPIM (La Plata Ionospheric)

Centro de cálculo Usuario

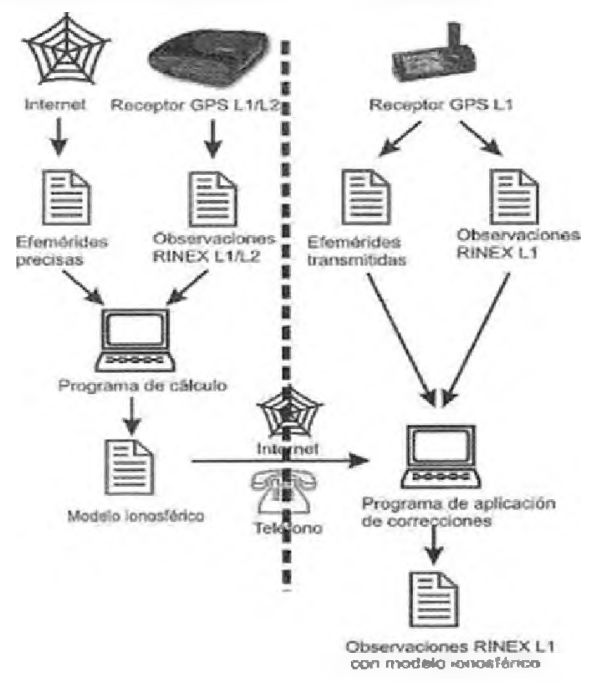

Figura 10. Formatos

de intercambio 


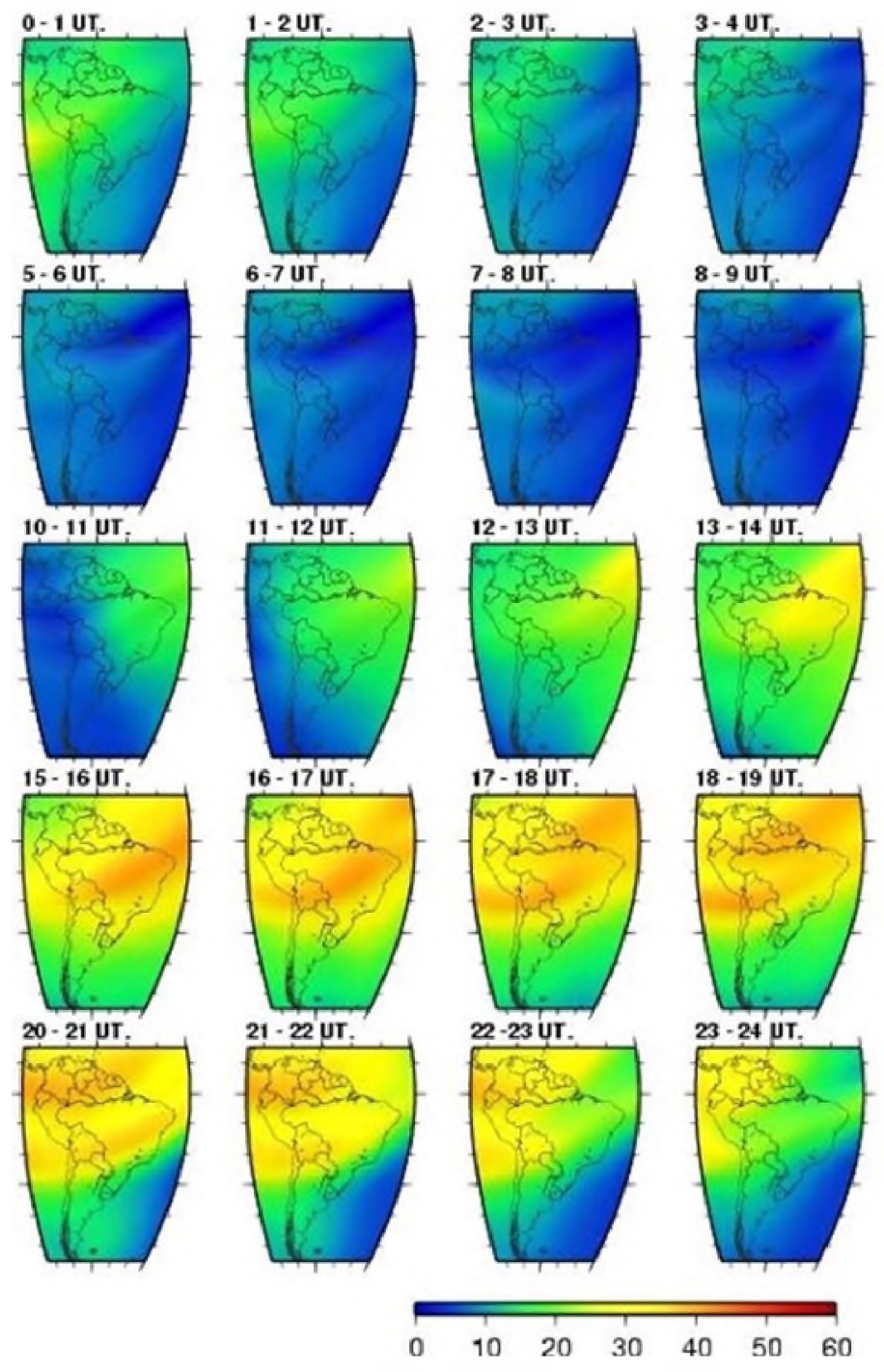

Figura 9. LPIM (La Plata Ionospheric)

\section{Bibliografía}

- AZPILICUETA, F. Modelos ionosféricos globales derivados de observaciones GPS y altimetría satelital, Tesis Doctoral, FCAG, UNLP, Noviembre de 2006.

- AZPILICUETA, F., BRUNINI, C. Analysis of the bias between TOPEX and GPS vTEC determinations, Journal of Geodesy, DOI 10.1007/s00190-008-0244-7, 2008.
- BRUNINI, C., MEZA, A. Y GENDE, M. Utilización de mediciones GPS para el análisis de la ionosfera: Aplicaciones geodésicas y geofísicas. En A. Introcaso (ed.), Contribuciones a la Geodesia en la Argentina de fines del siglo XX, homenaje a Oscar $\mathrm{Pa}$ rachu, ISBN 950-673-201-9, pp. 187-213, UNR Editora, 1999. 
- BRUNINI C., A. MEZA AND M. A. VAN ZELE, Análisis de confiabilidad entre mapas que describen el contenido vertical de electrones en la ionosfera, 20va reunión de la Asociación Argentina de Geofísicos y Geodestas, San Juan, 2000

- BRUNINI, C., CAMILION, E., AZPILICUETA, F. Simulation study of the influence of the ionospheric layer height in the thin layer ionospheric model, Journal of Geodesy, doi 10.1007/s00190-011- 0470-2, 2011.

- BRUNINI, C., CONTE, J. F., AZPILICUETA, F., BILITZA, D. A different method to update monthly median $\mathrm{hmF} 2$ values. Advances in Space Research (2013).

- BRUNINI, C., AZPILICUETA, F., NAVA, B., GULARTE, E. Global maps of montlymean values of the ionospheric F2-peak parameters derived from COSMIC / FORMOSAT-3, Jornal of Geodesy, en prensa (2013b).

- HOFMANN-WELLENHOF, et al. (1997).

Global Positioning System, Theory and practice. Springer-Verlag, Wien, New York.

- HUERTA E, JIMÉNEZ B, MANGIATERRA A, NOGUERA G, et al. (2001). Proyecto 19/1077 - Estación permanente GPS. Serie "Temas de Geociencia", № 7:
"Georreferenciación". UNR Editora, Rosario.

- HUERTA E, JIMÉNEZ B, MANGIATERRA A, NOGUERA G. GPS Posicionamiento Satelital.UNR Editora. Editorial de 1a Universidad Nacional de Rosario. 2005

- LEICK A. (1995). GPS satellite surveying. Wiley and Sons, New York.

- MANGIATERRA A, NOGUERA G, et al. (1999). Contribuciones a la geodesia en la Argentina de fines del siglo XX, Homenaje a Oscar Parachú. UNR Editora, Rosario.

- PALACIOS CID R, et al. (1999) Geodesia geométrica, física y por satélites. Editorial Colegio de Ingenieros Técnicos en Topografia, Madrid.

- SEEBER G. (1993). Satellite Geodesy. Walter de Gruyter, Berlin, New York.

- TEUNISSEN P, KLEUSBERG A. GPS for Geodesy. Springer, Berlin, New York.

- TORGE W. (2001). Geodesy. Walter de Gruyter, Berlin, New York. (1998)

- TORGE W. Geodesy. Walter de Gruyter, Berlin, New York.(2001)

- VALBUENA DURÁN J. L.,NúÑ̃EZGARCÍA DEL POZO A, et al. (1992). GPS, la nueva era de la topografía. Ediciones Ciencias Sociales SA, Madrid.

- ZAKATOV P. Curso de geodesia superior. Editorial Mir, Moscú (1981) 